\section{Obst und Gemüse gegen Brustkrebs?}

Wer früh auf eine ballaststoffreiche Ernährung setzt, reduziert möglicherweise sein Brustkrebsrisiko. Das lässt eine Auswertung der laufenden US-amerikanischen Nurses' Health Study II vermuten. Von fast 44.300 Teilnehmerinnen standen Informationen über die Aufnahme von Ballaststoffen als Heranwachsende zur Verfügung. Wie Forscher von der Harvard Medical School in Boston berichten, hatten Frauen pro Aufnahme von täglich $10 \mathrm{~g}$ Ballaststoffen in der Jugend ein um 14\% verringertes Brustkrebsrisiko. Als mögliche Erklärung nennen die Autoren u.a. eine verbesserte Insulinsensitivität. Außerdem könnten Ballaststoffe die Plasmaspiegel an Östrogen senken.

Pediatrics 2016, online 1. Februar 2016

\section{Kartoffeln \\ - ein Risiko für Schwangere?}

Glaubt man US-Epidemiologen, sollten Schwangere Kartoffeln meiden. Denn der Konsum der Stärkefrucht steigere das Risiko für einen Gestationsdiabetes. Die Rate war bei einer Portion pro Woche um 20\% erhöht, bei zwei bis vier Portionen um $27 \%$ und bei mehr als fünf um $50 \%$, verglichen mit unter einer Portion. Die Daten entstammen der Nurses' Health Study.

Bao W et al. BMJ 2016; 352:h6898

\section{Fisch hält das Gehirn frisch}

Meeresfische sind zwar reich an Quecksilber. Trotzdem ist eine fischreiche Ernährung für das Gehirn wohl von Vorteil. Forscher aus Chicago untersuchten die Gehirne von 286 verstorbenen Teilnehmern des prospektiven Memory and Aging Projects. Darin fahndeten sie nach Markern für eine Alzheimerdemenz (AD) und bestimmten den Quecksilbergehalt. Aus früher erhobenen Daten wusste man, wie häufig die Verstorbenen zu Lebzeiten Fisch verzehrt hatten. Wie sich zeigte, war die Quecksilberkonzentration im Gehirn umso höher, je öfter die Teilnehmer Fisch gegessen hatten. Zugleich ließen sich aber bei hohem Fischkonsum seltener Plaques und Fibrillen nachweisen - zumindest bei Trägern des Alzheimer-Risiko-Allels ApoE4. Eine AD-Pathologie war bei ihnen um $47 \%$ seltener als bei Fischverächtern.

JAMA 2016;315(5):489-497

\title{
Schmerzmanagement verbessern
}

Schmerzen bedeuten eine erhebliche Einschränkung von Lebensqualität. Deshalb zählt ein angemessenes Schmerzmanagement zu den zentralen Aufgaben von Pflegenden. Doch die Schmerzversorgung von Pflegeheimbewohnern ist nachweislich inadäquat. Um diesem Defizit zu begegnen, untersuchte ein Expertenteam der Berliner Charité Fortbildungsmethoden für Pflegende und entwickelte neue Handlungsempfehlungen.

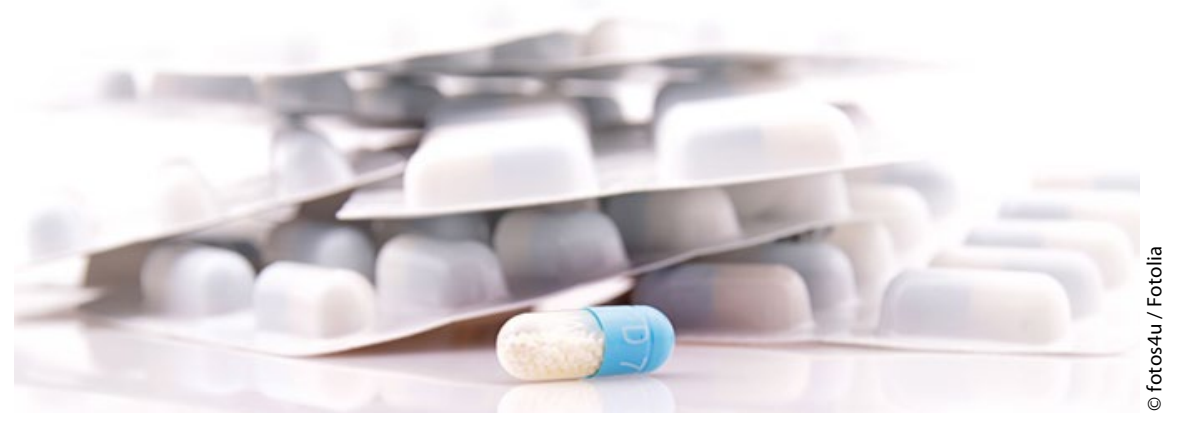

m Projekt „PAIN-Intervention“ wurden für die Interventionsgruppe Pflegepersonal ein Ganztagsworkshop und eine Onlinefortbildung für Hausärzte konzipiert und durchgeführt. Dabei wurden die Innovation als Gegenstand der Veränderung, das soziale System, in dem Veränderungsprozesse stattfinden und die Zeit als beeinflussende Faktoren gemäß der Diffusionstheorie von Rogers besonders berücksichtigt. Auch das Wissen der Beteiligten und ihre Überzeugung bezüglich der Neuerung spielten hinsichtlich der Annahme und Umsetzung der Innovation „Schmerzmanagement" eine bedeutsame Rolle. In einer Kontrollgruppe wurde eine 45-minütige Basisveranstaltung zur Schmerzthematik für Pflegende sowie eine Informationsveranstaltung für Hausärzte angeboten.

Der interaktive Ganztagsworkshop wurde in zwölf Pflegeheimen eines Trägers durchgeführt und vermittelte nicht nur fachliches Wissen für examinierte Pflegende, sondern reflektierte vor allem eigene Haltungen und Einstellungen der Teilnehmenden sowie die Förderung des bewussten Umgangs mit dem Thema Schmerz. Die Basisinformation für die Kontrollgruppe wurde in Form einer Präsentation durchgeführt und vermittelte hauptsächlich Wissen zur Optimierung des Schmerzmanagements, mit dem Lernziel die Fachkompetenzen zu steigern und vorhandenes Wissen zu reaktivieren.

Mittels standardisierter Fragebögen konnten folgende Ergebnisse zur Evaluation der Fortbildungen gesichert werden: Beide Veranstaltungen wurden als sehr zufriedenstellend bewertet und in beiden Gruppen sehen sich die Pflegenden in einer bedeutenden Rolle im Rahmen des Schmerzmanagements. Signifikante Unterschiede zugunsten des Ganztagsworkshops zeigten sich insbesondere bei der Bewertung der Austauschmöglichkeiten, der erlebten Sicherheit im Umgang mit den Schmerzen der Bewohnerinnen und bezüglich der Information über nichtmedikamentöse Maßnahmen.

Folglich nehmen Methodik und zeitlicher Rahmen sowie der damit verbundene Umfang der Inhalte Einfluss auf die Bewertung. Auch die hohe Relevanz einer multiperspektivischen Umsetzung von Fortbildung wurde deutlich. Die Autoren bewerten eine handlungsorientierte und facettenreichen Wissensvermittlung positiv, zur erfolgreichen Implementierung von Innovationen sind jedoch weitere Maßnahmen, vor allem zum bewussten Umgang mit (neuem) Wissen, notwendig.

Kissel-Kröll, A., Budnick, A., Könner, F. et al. Evaluation einer Fortbildung für Pflegende zum Schmerzmanagement in Pflegeheimen. HeilberufeSCIENCE (2016) 1, 9-18 